\title{
Comparison of Shear Bond Strength of Ceramic Brackets Bonded To Ceramic Surface - An In-Vitro Study
}

Devyani Ninad Thorat ${ }^{1}$

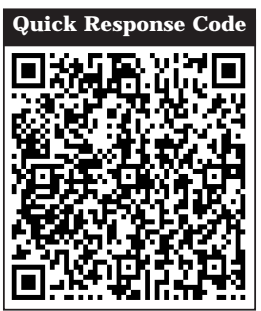

doi : $10.5866 / 2016.8 .10148$

Resident, Department of

Orthodontics and Dentofacial Orthopaedics

Rajiv Gandhi University of Health Sciences, Bangalore

\section{Article Info:}

Received: J uly 11, 2016

Review Completed: August 10, 2016

Accepted: September 9, 2016

Available Online: September, 2016 (www.nacd.in) (c) NAD, 2016 - All rights reserved

\section{Email for correspondence:}

Ddv5678@gmail.com

\begin{abstract}
:
Background: In recent years there has been an increase in the number of adults seeking orthodontic treatment and most of the adult patients usually present with restored teeth. Dental ceramics is widely used to restore missing and damaged teeth. Therefore, the Orthodontist is often faced with the challenge of effectively bonding orthodontic brackets to ceramic restorations in adult patient.
\end{abstract}

Objectives: To compare the shear bond strength between ceramic brackets and ceramic surface with three different surface treatments.

Materials \& Methods: Thirty ceramic facets were prepared with a proper thickness of $2 \mathrm{~mm}$, resembling maxillary central incisor labial surface. These facets were then divided into 3 groups $(n=10)$ i.e. Group 1: Treated with 9\% HFA \& silane, Group 2: sandblasted \& silane, Group 3: 37\% Phosphoric acid \& silane. Thirty ceramic brackets were bonded on these facets as per manufacturer's instructions. These brackets were then de-bonded by universal testing machine to measure the shear bond strength values. The evaluation of the variation of the shear bond strength (SBS) among test groups was done using one-way ANOVA test and interexperimental group comparison was done by Newman-Keuls multiple post hoc test.

Results: Group I (20.39 55.19MPa) showed the highest bond strength followed by Group II (15.42+3.65MPa) and Group III $(14.30 \pm 2.55 \mathrm{MPa})$. Statistically significant difference existed between group I \& group II, group I \& group III except group II \& group III.

Conclusion: Shear bond strength of ceramic brackets bonded to ceramic surfaces with all the three surface conditioning methods were adequate. On comparison of the three surface conditioning methods, 9\% Hydrofluoric acid showed highest shear bond strength followed by Sandblasting and 37\% Phosphoric acid.37\% Phosphoric acid is recommended since it has adequate bond strength. It is least harmful to oral mucosa as compared to $9 \%$ Hydrofluoric acid and less cumbersome procedure when compared to Sandblasting.

Key words: Shear bond strength, Ceramic.

\section{INTRODUCTION}

In recent years there has been an increase in the number of adults seeking orthodontic treatment and most of the adult patients usually present with restored teeth. Dental ceramics is widely used to restore missing and damaged teeth. ${ }^{1}$ Ther efore, the orthodontist is often faced with the challenge of effectively bonding orthodontic brackets to ceramic restorations in adult patient. ${ }^{2}$ Ceramic is an inert material, therefore ceramic surfaces do not bond 
readily with orthodontic brackets, hence the surface characteristics of ceramic are altered through certain approaches before bonding the brackets to ceramics. Some of these are applying silane coupling agents to the ceramic surface and etching with hydrofluoric acid, or sandblasting the ceramic surface. Phosphoric acid solution is also used to enhance bond strength of the ceramic surface but it is not efficient as microetching the ceramic surface with the application of hydrofluoric acid and silane couple. $^{3}$

Adult orthodontic patients wanting the most invisible treatment possible spurred the development of "transparent" brackets that provide an aesthetic solution. ${ }^{4}$ The bonding process of ceramic brackets to enamel is identical to that of stainless steel brackets, but, occasionally in adults these brackets must be bonded to ceramic restoration or crowns. ${ }^{4}$

Pre-treatment of ceramic surfaces is necessary to obtain sufficient strength to bond orthodontic brackets to all ceramic restorations. Several options have been described which are generally combinations of various mechanical and chemical conditioning methods, such as bonding to glazed ceramic with coupling agent (silane), deglazing the ceramic by roughening the surface (diamond burs; air particle abrasion (APA) with aluminium oxide), and chemical preparation of the ceramic with acids, such as phosphoric, hydrofluoric, acidulated phosphate fluoride. ${ }^{2}$ These methods achieved bond strengths equivalent to bonding to enamel etched with $37 \%$ phosphoric acid.

It has been shown that silane coupling agent appear to enhance the bond strength by increasing the chemical bond between resin composite and ceramic material. In general, the silane coupling agent is applied with chemical and mechanical roughening procedures. However the harmful effects of hydrofluoric acid on the soft tissue has been highlighted. $^{2}$

Mechanical roughening with APA has been shown to provoke crack initiation on the ceramic surface. However in order to obtain a viable bond between the orthodontic brackets and the ceramic surface, mechanical or chemical roughening is inevitable. $^{2}$

Though some of the methods have gained significant acceptable bond strength, currently no method has been proven to be most effective. This prompted us to undertake a study where comparison of the shear bond strength of ceramic bracket bonded to ceramic surface was carried out and evaluated which is the most effective and reliable bonding procedure when bonding of ceramic brackets with ceramic surface is to be carried out in adult patients with aesthetic concern .

\section{MATERIALS AND METHODS:}

RESEARCH DESIGN: This is an experimental in vitro study carried out at Department of Orthodontics.

\section{METHOD OF STUDY:}

1. Thirty ceramic facets (Ivodar vivadent P-300, IPS classic) were prepared with a proper thickness of $2 \mathrm{~mm}$, resembling maxillary central incisor labial surface.

2. These facets were then attached with a metal casting handle, so that support was provided for the facets to be embedded into dental plaster (Figure 1).

3. The metal casting handle was mounted into the dental plaster vertically to cover upto the usual level of the alveolar bone around the incisor tooth.

4. Facets were aligned with the facial surface of the tooth perpendicular with the bottom of the mould

5. Thirty facets were then divided into 3 groups each consisting of 10 ceramic facets each (Figure 4)

6. Group 1- treated with $9 \%$ hydrofluoric acid and silane (90 seconds) Group 2- treated with sandblasting (50-120 $\mu \mathrm{m})$ and silane Group 3treated with $37 \%$ phosphoric acid and silane (60 seconds)

7. Ceramic brackets were then bonded to each conditioned ceramic facets with light cure composite resin, and then light cured with light curing unit (Woodpecker LED.D,420-480nm) for 40 seconds each

8. The specimens were then stored in water bath at $37^{\circ}$ for 24 hours.

9. Specimens werethen tested on universal testing machine for shear force required to debond the brackets (Figure 5 and 6 )

10. The shear bond strength data was then subjected to ANOVA test 


\section{BONDING PROTOCOL}

The bonding protocol following the manufacturer's instructions was carried out. All 30 incisor facets were conditioned with 3 different procedures. Later silane coupling agent was applied to each conditioned surface with a mini brush tip and allowed to evaporate for 60 seconds, if not completely dry after 60 seconds then air dried with oil free air. Bonding agent (Transbond XT primer) was then applied with a mini brush on the dried silane surface and cured for 20 seconds. Ceramic brackets were then bonded to each conditioned ceramic facet using light cure composite resin (Transbond XT, 3M Unitek) and light cured for forty seconds each (Figure 2 and 3). Before light curing the adhesive, the brackets were pressed against the tooth and then excess adhesive was removed with sharp explorer. After bonding all the ceramic facets with the ceramic brackets i.e. the 30 specimens were stored in water at $37^{\circ}$ for 24 hours.

\section{FINAL DEBONDING}

A customised jig was suspended from the crosshead of a universal testing machine (TUE-C400, Fine Spavy Associated \& Engineers Pvt Ltd, Miraj). A gingivo-occlusal load was applied to the bracket, producing shear force at the bracket-facet interface for all the three groups. A computer, electronically connected with the test machine, recorded the results of each test. Shear bond strengths were measured at a crosshead speed of $0.5 \mathrm{~mm} / \mathrm{min}$ (Figure 5 and 6 )

The force required to break the bracket-facet bond was recorded in kilo Newtons $(K n)$ and converted to megapascals ( $\mathrm{Mpa}$ ) using the surface area of the bracket base. The following equation was used for the conversion:

Stress $(\mathrm{Mpa})=\frac{\text { Force }(\mathrm{kN})}{\text { Area }(\mathrm{mm} \text { square })} \times 10^{3}$

Area: surface area of the ceramic bracket base

Surface area of the ceramic bracket base was 15.75 $\mathrm{mm}^{2}$

\section{DATA ANALYSIS:}

The comparison of the shear bond strength of the three test groups were evaluated statistically using ANOVA test. Pairwise comparison between the test groups was done by Newman-Keuls post hoc analysis. A probability value ( $P$ value) 0.05 was considered to be statistically significant.

\section{RESULTS:}

The findings of the present study are tabulated (Table 1-4):

\section{DISCUSSION}

The Orthodontists' understanding of the demand for aesthetic improvement in bracket appearance influences patient acceptance of orthodontics and patients current access to vast amounts of public information. Patients want to feel and look as healthy as possible, and they are becoming increasingly influenced by esthetics and demanding to be treated with esthetics in mind. Therefore ceramic brackets are widely used. ${ }^{5-7}$ Ceramic restorations such as ceramic crowns or veneers are most commonly present in adult patients..$^{8-11}$ Therefore often the orthodontist must bond the ceramic brackets to ceramic restorations. The bonding force of ceramic brackets is achieved by mechanical retention of the adhesive to bracket base and by using silane coupling agent after preparation of the porcelain surface.

Since glazed porcelain surface are not amenable to resin penetration for orthodontic bonding, mechanical or chemical pre-treatments of the surface is essential for successful direct bonding to porcelain. Although various surface treatment methods have been suggested each one has some disadvantages and limitations. Mechanical roughening with sandblasting is reported to provoke crack initiation within the ceramic. On the other hand, hydrofluoric acid has been found to be a harmful and irritating compound for soft tissue. Organosilane coupling agents are suggested to enhance bonding brackets to porcelain surfaces, but they fail to provide clinically sufficient bond strengths when used alone. To improve bond strength combination of methods are recommended. ${ }^{8}$

The present study was conducted to determine the shear bond strength of ceramic brackets bonded to ceramic surfaces with different surface treatments. This was required to know the best possible surface treatment to achieve optimal bond strength between ceramic brackets and ceramic surface.

In the present conducted experimental in-vitro study, thirty ceramic facets were divided into three groups $(n=30)$.

GROUP 1: ceramic facets treated with $9 \%$ hydrofluoric acid (90 seconds) and silane 


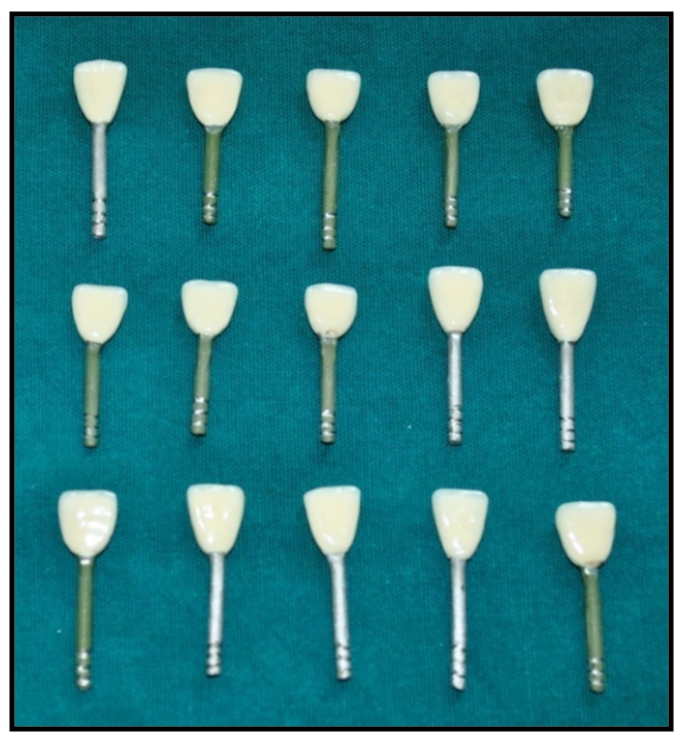

Figure 1: Thirty ceramic facets (including both the surfaces) with casting handle.

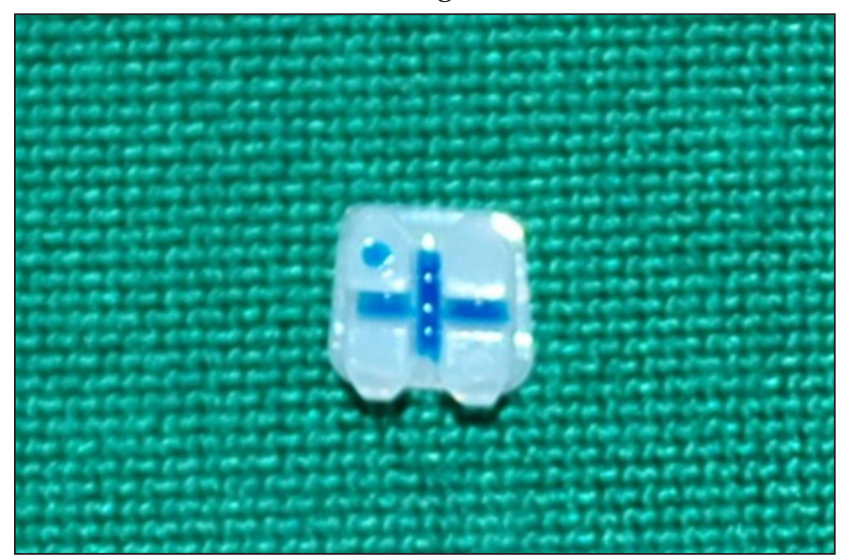

Figure 3: Clarity advanced central incisor ceramic bracket (3M Unitek)

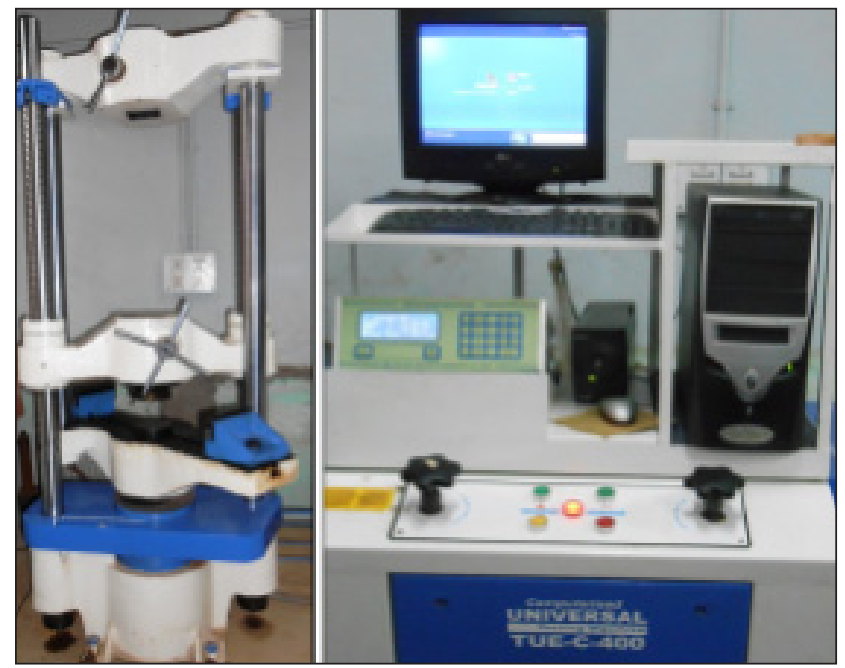

Figure 5: Universal testing machine set up

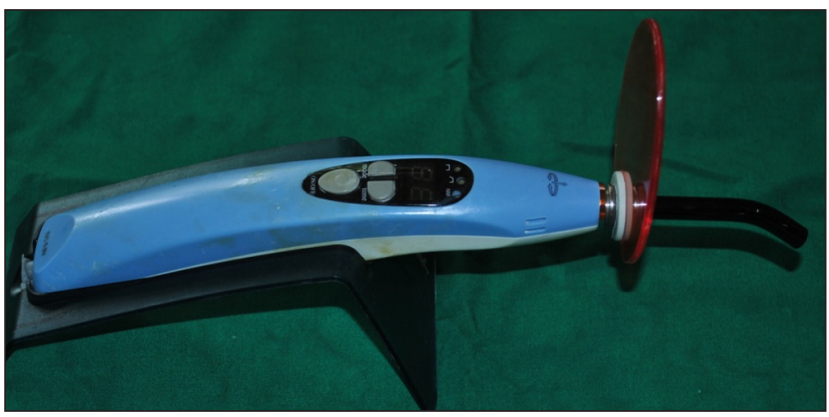

Figure 2: Light curing unit (Woodpecker) used in the present study.

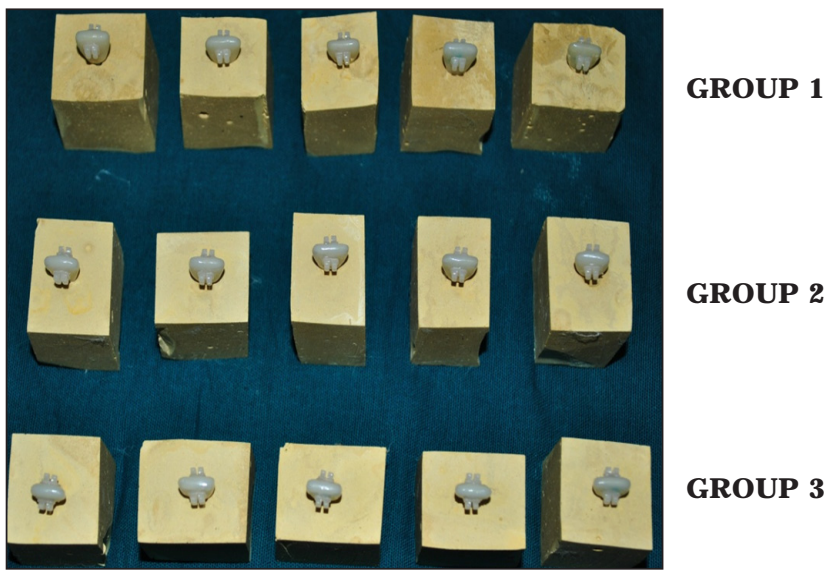

Figure 4: Samples consisted of three test groups (10 in each Group). Group 1 - Treated with 9\% Hydrofluoric acid and silane; Group 2 - Treated with sandblasting with aluminium oxide particles and silane; Group 3 - Treated with $37 \%$ phosphoric acid and silane.

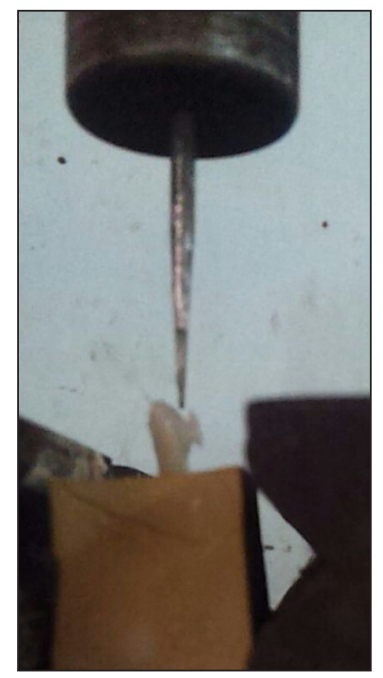

Figure 6: Customized jig producing shear force at the bracket- ceramic facet interface 
Table 1: Individual Shear bond strengths (MPa) of the ten samples of each group

\begin{tabular}{lccc}
$\begin{array}{c}\text { SI. } \\
\text { No. Hydrofluoric } \\
\text { acid }\end{array}$ & $\begin{array}{c}\mathbf{3 7 \%} \\
\text { Phosphoric } \\
\text { acid }\end{array}$ & Sandblasted \\
\hline 1. & 24.1 & 17.7 & 12.6 \\
\hline 2. & 24.1 & 16.5 & 12.6 \\
\hline 3. & 25.3 & 11.4 & 15.2 \\
\hline 4. & 10.1 & 15.8 & 12.6 \\
\hline 5. & 22.8 & 17.7 & 19.0 \\
\hline 6. & 20.3 & 13.9 & 15.2 \\
\hline 7. & 25.3 & 12.6 & 22.8 \\
\hline 8. & 15.2 & 11.4 & 12.6 \\
\hline 9. & 15.2 & 14.6 & 12.6 \\
\hline 10. & 21.5 & 11.4 & 19.0 \\
\hline & & &
\end{tabular}

Table 2: Mean, Sandard Deviation, standard error and coefficient of variation of shear bond strengths (Mpa) in three test groups

\begin{tabular}{lcccc} 
Groups & Mean & SD & SE & CV \\
\hline 9\% Hydrofluoric acid & 20.39 & 5.19 & 1.64 & 25.46 \\
\hline Sandblasted & 15.42 & 3.65 & 1.15 & 23.68 \\
\hline 37\% Phosphoric acid & 14.30 & 2.55 & 0.81 & 17.84 \\
\hline
\end{tabular}

Table 3: Pair wise comparison of three groups (9\% Hydrofluoric acid, $37 \%$ phosphoric acid and Sandblasted) with respect to shear bond strength by Newman-Keuls multiple post hoc procedures A) $\mathbf{9 \%}$ Hydrofluoric acid VS $37 \%$ Phosphoric acid

\begin{tabular}{lcc} 
Groups & $\begin{array}{c}\mathbf{9} \% \text { Hydrofluoric } \\
\text { acid }\end{array}$ & $\begin{array}{c}\mathbf{3 7 \%} \\
\text { Phosphoric acid }\end{array}$ \\
\hline Mean & 20.39 & 14.3 \\
\hline $\begin{array}{l}\text { Standard } \\
\text { deviation }\end{array}$ & 5.19 & 2.55 \\
\hline$P=0.0052 *$ & & \\
\hline
\end{tabular}

\begin{tabular}{|c|c|c|}
\hline Groups & $\begin{array}{c}\mathbf{9 \%} \text { Hydrofluoric } \\
\text { acid }\end{array}$ & Sandblasted \\
\hline Mean & 20.39 & 15.42 \\
\hline $\begin{array}{l}\text { Standard } \\
\text { deviation }\end{array}$ & 5.19 & 3.65 \\
\hline
\end{tabular}

$\mathrm{P}=0.0092^{*}$

B) $9 \%$ Hydrofluoric acid VS Sandblasting

\begin{tabular}{lcc} 
Groups & $\begin{array}{c}9 \% \text { Hydrofluoric } \\
\text { acid }\end{array}$ & $\begin{array}{c}\text { Sandblasted } \\
\text { Mean }\end{array}$ \\
\hline $\begin{array}{l}\text { Standard } \\
\text { deviation }\end{array}$ & 2.55 & 15.42 \\
\hline$P=0.5314$ & 3.65 \\
\hline C) $37 \%$ Phosphoric acid VS Sandblasting
\end{tabular}

TABLE 4: Comparison of three groups (9\% Hydrofluoric acid, Sandblasted and 37\% Phosphoric acid) with respect to shear bond strength by one way ANOVA. *P $<0.05$ is considered statistically significant.

\begin{tabular}{lccccc}
$\begin{array}{l}\text { Source of } \\
\text { variation }\end{array}$ & $\begin{array}{c}\text { Sum of } \\
\text { squares }\end{array}$ & $\begin{array}{c}\text { Degrees of } \\
\text { freedom }\end{array}$ & $\begin{array}{c}\text { Mean sum } \\
\text { of squares }\end{array}$ & F-value & P-value \\
\hline Between groups & 210.14 & 2 & 105.07 & 6.7372 & $0.0042^{*}$ \\
\hline Within groups & 421.09 & 27 & 15.60 & & \\
\hline Total & 631.23 & 29 & & & \\
\hline
\end{tabular}

GROUP 2: ceramic facets sandblasted with aluminium oxide particles $(90 \mu \mathrm{m})$ and silane

GROUP 3: ceramic facets treated with $37 \%$ phosphoric acid (60 seconds) and silane.

The determination of the shear bond strength was done using universal testing machine at crosshead speed of $0.5 \mathrm{~mm} / \mathrm{min}$
The mean and standard deviation of the shear bond strength values obtained for each specimen of the three groups have been given in the table 2 and graph 1 . The mean and standard deviation values of shear bond strength for test groups are

GROUP 1: 20.39 $\pm 5.19 \mathrm{MPa}$

GROUP 2: $15.42 \pm 3.65 \mathrm{MPa}$ 


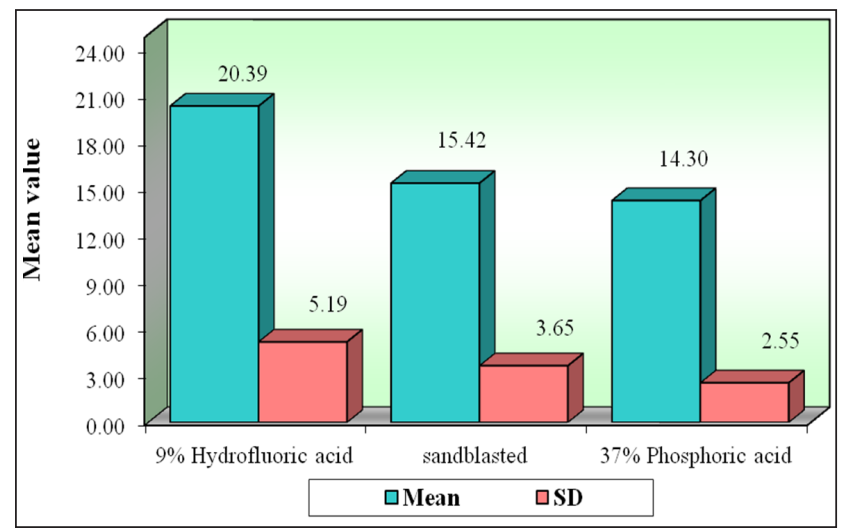

Graph 1: Comparison of three groups (9\% Hydrofluoric acid, Sandblasted and 37\% Phosphoric acid) with respect to shear bond strength.

\section{GROUP 3: 14.30 \pm 2.55 MPa respectively.}

Mean and standard deviation of shear bond strength between group 1, group 2 and group 3 were compared statistically by one-way ANOVA test. The results in the Table 4 showed highly significant difference between the groups with $\mathrm{F}$-value (6.7372) and $P$ value $(0.0042)$.

Pairwise comparison test (newman keuls test) of shear bond strength (SBS) values between the three test groups showed that the comparison between the $9 \% \mathrm{HFA}$ and sandblasting, 9\%HFA and 37\% Phosphoric acid was significant (Table 3-B \& A). However the shear bond strength values did not show significant difference between sandblasting and 37\% Phosphoric acid groups (Table 3 - C).

The shear bond strength between ceramic brackets and ceramic facets treated with $9 \%$ hydrofluoric acid is statistically higher than the other two groups (Graph 1). Although the other two groups also showed bond strengths higher than the optimal bond strength required for bonding the ceramic brackets showed a tendency to break off during debonding procedure which were treated with $9 \% \mathrm{HF}$. The brackets initially chipped off and then debonding was carried out. This showed that the bond strength with $9 \%$ hydrofluoric acid group was higher.

In the present study, the highest bond strength was obtained for group 1 i.e treated with $9 \% \mathrm{HF}$ and silane coupling agent. This is consistent with the observations made by Hakan Tukkahraman and $\mathrm{H}$. Cenker Kucukesme (2006) who conducted a similar study, finally found that surface treatment with HFA and silane coupling agent produced the highest bond strength. ${ }^{8}$ Conflicting issues exist when hydrofluoric acid and silane are used together. Kocadereli et al (2001) conducted a similar study which showed that porcelain surface conditioning with hydrofluoric acid etching followed by silane application resulted in higher bond strengths. Therefore in the present study silane application was recommended to bond ceramic bracket to porcelain surface to achieve bond strengths that are clinically acceptable. ${ }^{5}$ In contrast, Schmage et al (2003) conducted a similar study and showed that highest bond strength values were obtained with sandblasting and silicatization with silane or HFA without silane. Therefore it was concluded that the use of silane after HFA etching did not increase the bond strength. ${ }^{6}$ In the present study, the highest SBS were obtained with HFA and silane. This is an expected result as HFA facilitates micro-mechanical retention and silane provides a chemical link between porcelain and composite resin. The contradictory results may be explained by the differences in storage conditions, bonding agents and ceramic types. ${ }^{8}$

Considering the harmful and irritating effects of etching with HFA (J ochen 1973, Moore and Manor 1982) and also the time consuming procedure and crack initiation when using sandblaster for sandblasting, we could consider surface conditioning of ceramic surface with $37 \%$ phosphoric acid as an optimal and safe surface treatment as it is commonly used in daily routine bonding of orthodontic brackets with enamel surface. ${ }^{8}$

The optimal bond strength required for orthodontic clinical use is as yet unknown. Reynolds in 1975 suggested that for an adhesive system to have acceptable clinical performance, in vitro bond strength of 5.9- 7.8 $\mathrm{MPa}$ is required. ${ }^{12-17} \mathrm{He}$ also suggested that a minimum bond strength of 6 to 8 $\mathrm{M} \mathrm{pa}$ is adequate for most clinical orthodontic needs. ${ }^{9}$ The present study shows that shear bond strength of all three groups is higher than the optimal range required for bonding.

The nature of the forces directed onto orthodontic brackets in the mouth is likely to be a combination of shear, tensile and torsion. ${ }^{18}$ The bond strength of bracket adhesive - porcelain system in orthodontic bonding varies and depends on factors such as the type of adhesive, bracket base design, storage media, type of porcelain, appliance force systems and the clinician's technique. While using 
the universal testing machine, it is not practically possible to simulate debonding forces that act in an in-vivo set-up and the same goes for the rate of loading for the testing machine. Testing a single debonding force eg: shear force will beeasier for such type of a study design. ${ }^{4}$ These are a few among the many factors, which may contribute to the variability and difference of opinion among researchers regarding the clinically acceptable bond strength. However, in the present study just shear forces were evaluated.

\section{CONCLUSION}

Shear bond strength of ceramic brackets bonded to ceramic surface with all the three surface conditioning methods were adequate. On comparison of the three surface conditioning methods 9\% Hydrofluoric acid showed higher shear bond strength followed by Sandblasting and 37\% Phosphoric acid. The recommended conditioner is $37 \%$ phosphoric acid as it has adequate bond strength, least harmful to the oral mucosa on comparison to $9 \%$ hydrofluoric acid and is less cumbersome procedure as compared to sandblasting.

\section{BIBLIOGRAPHY}

1. Abu Alhaija E, Abu ALReesh I, ALWahadni A. Factors affecting the shear bond strength of metal and ceramic brackets bonded to different ceramic surfaces. Eur J Orthod 2010; 32:274-80.

2. Turk T, Sarac D, Sarac YS, Turk SE. Effects of surface conditioning on bond strength of metal brackets to allceramic surfaces. Eur J Orthod 2006; 28:450-6.

3. Bilgic $F$, Alkis $H$, Gungor AY, Tuncdemir AR, Malkoc MA. Shear bond strength of ceramic brackets bonded to three different porcelain surfaces. Eur J Prostho 2013; 1(1):1720.

4. Harari D, Shapira-Davis S, Gillis I, Roman I, Redlich M. Tensile bond strength of ceramic brackets bonded to porcelain facets. Am J Orthod Dentofacial Orthop 2003; 123:551-4.

5. Kocadereli I,Canay S, Akca K. Tensile bond strength of ceramic orthodontic brackets bonded to porcelain surfaces. Am J Orthod Dentofacial Orthop 2001; 119:617-20.

6. Schmage P, Nergiz I, Herrmann W, Ozcan M. Influence of various surface conditioning methods on the bond strength of metal brackets to ceramic surfaces. Am J Orthod Dentofacial Orthop 2003; 123:540-6.

7. Soderquist SA, Drummond J L, Evans CA. Bond strength evaluation of ceramic and stainless steel bases subjected to cyclictensileloading. AmJ Orthod Dentofacial Orthop 2006; 129:175.e7-175.e12.

8. Turkkahraman $\mathrm{H}$, Kucukesmen HC. Porcelain surface conditioning techniques and shear bond strength of ceramic brackets. Eur J Orthod 2006; 28:440-3.

9. Maryanchik I, Brendlinger E, Fallis D, VandewalleK. Shear bond strength of orthodontic brackets bonded to various esthetic pontic materials. Am J Orthod Dentofacial Orthop 2010; 137:684-9.

10. Akhoundi A, Kamel MR, Hashemi M, I mani M. Tensilebond strength of metal brackets bonding to glazed ceramic surfaces with different surface conditionings. J of Dentistry, Tehran university of medical sciences, Tehran, I ran 2011; 8(4):201-8.

11. Faltermeier A, Roemer $\mathrm{P}$, Reicheneder $\mathrm{C}$, Proff $\mathrm{P}, \mathrm{K}$ linke $\mathrm{T}$. The influence of surface conditioning of ceramic restorations before metal bracket bonding. Material Sciences and Applications 2012; 3:1-5 .

12. Kukiattrakoon B, Samruajbenjakul B. Shear bond strength of ceramic brackets with various bracket base designs bonded to aluminous and fluorapatite ceramics. Eur J Orthod 2010; 32:87-93.

13. Singh S, Raghav P, Gaur A, Reddy M, Mishra V. Bonding to a porcelain surface: factors affecting the shear bond strength. APOS trends in orthodontics 2013; 3(5):152-6.

14. Bayram M, Yesilyurt C, Kusgoz A, Ulker M, Nur M. Shear bond strength of orthodontic brackets to aged resin composite surfaces: effect of surface conditioning. Eur J Orthodontics 2011; 33:174-9.

15. Uysal T, Ustdal A, Kurt G. Evaluation of shear bond strength of metallic and ceramic brackets bonded to enamel prepared with self-etching primers. Eur J Orthod 2010; 32(2):214-8.

16. Girish PV, Dinesh U, Bhat R, Shetty PC. Comparison of shear bond strength of metal brackets bonded to porcelain surface using different surface conditioning methods: An in vitro study. J Contemp Dent Pract 2012; 13(4):487-3.

17. Reynolds I R. A review of direct orthodontic bonding. B J Orthodontics 1975; 2:171-8.

18. Greenlaw R, Way DC, Galil KA. An in vitro evaluation of a visible light-cured resin as an alternative to conventional resin bonding systems. Am J Orthod Dentofacial Orthop 1989; 96(3):214-20.

\section{Gain quick access to our journal online View our journal at www.nacd.in}

